\title{
Among mesenchymal stem cells: for the best therapy after ischemic stroke
}

\author{
Yuka lkegame*1,2 \\ See related research by Gutiérrez-Fernández et al., http://stemcellres.com/content/4/1/11
}

\begin{abstract}
Owing to the several advantages that they have over other cells, mesenchymal stem cells are among the most promising tools in stem cell therapy after ischemic stroke. Mesenchymal stem cells can be obtained from various sources. Therefore, a wide range of careful comparative studies of these cells is required, from the preclinical stage (in vitro and in vivo) to the clinical stage, in order to develop the best therapy with the most appropriate cell type. An in vivo study in this issue of Stem Cell Research \& Therapy compares the therapeutic potential of two cell types, obtained from bone marrow and adipose tissue, in a rodent stroke model. This commentary discusses the significance of comparative studies of mesenchymal stem cells, including the related article in translational research.
\end{abstract}

\section{Mesenchymal stem cells in investigations for stem cell therapy after stroke}

Stem cell therapy is one of the most anticipated emerging therapies in the treatment of ischemic stroke. A variety of stem cells or progenitor cells, including neural stem cells, CD34 ${ }^{+}$cells, and mesenchymal stem cells (MSCs), have been investigated with respect to their therapeutic potential in a number of preclinical studies. Subsequently, clinical studies also have been conducted on several cell types. In the search for an ideal cell delivery, a variety of conditions - such as cell quality (which cell type is used, age, and cell culture), the administration route, cell dose, timing, and cell modification - need to be examined. Which pathological conditions are suitable for each therapy also needs to be evaluated [1-4]. In this issue of Stem Cell Research \& Therapy, Gutiérrez-Fernández and colleagues [1] look for the best protocol for using MSCs

*Correspondence: ikegame-nsu@umin.ac.jp

'Department of Cell Signaling, Gifu University Graduate School of Medicine,

1-1 Yanagido, Gifu 5011194, Japan

Full list of author information is available at the end of the article and compare the therapeutic capacity of bone marrowderived MSCs (BM-MSCs) and adipose tissue-derived MSCs (AD-MSCs) as allogenic grafts intravenously administered in rodents in the acute phase with a permanent ischemic stroke model. The focal ischemia model is similar to thrombotic or embolic infarction in patients. The authors evaluate the treatment effects in both the acute phase and the subacute phase by analyzing neurological function, pathological changes, and histological brain repair [1].

MSCs are promising therapeutic tools for regenerative medicine. Within the last decade, several clinical trials that employ MSCs have been started, and some of them have shown positive safety results and effects [2,4]. MSCs can be obtained from diverse adult tissues (for example, bone marrow, adipose tissue, peripheral blood, and other mesoderm tissues). Furthermore, it is said that MSCs have more advantages in clinical application than other cell types; this includes such advantages as multilineage potential, immunomodulative ability, the secretion of trophic factors for brain tissue, and easier cell preparation with fewer ethical problems [4-6]. The immunological feature enables a recipient to avoid the risk of rejection without immunosupressants by producing immune tolerance, which means that MSCs can provide easier allogenic cell therapy free from demerits of immune suppressor to patients in the acute phase. An autologous graft has the least risk of side effects but, owing to the time required for cell expansion, is difficult to apply in the acute phase. Certainly, a few reports have shown that autologous BM-MSC administration in the subacute or later phase could also be effective [7], but given the ability of MSCs to product trophic factors, acute-phase therapy with an allogenic graft needs to be considered.

On the other hand, owing to the diversity of MSC sources, which type of MSC is more favorable is still quite controversial and will need to be clarified. Bone marrow and adipose tissue are thought to be among the most suitable sources for practical use, as both tissues are abundant enough to easily isolate the required number of MSCs. Gutiérrez-Fernández and colleagues [1] demonstrate that the two allogenic MSCs showed equivalent 
therapeutic benefits after permanent ischemia. One report indicates different brain-protective effects for BMMSCs and AD-MSCs in a transient ischemic stroke model [8]. However, if the two MSCs can contribute equally to a cure, an application of MSC therapy would increase by using the two most abundant cell sources.

\section{Mechanism analysis for neural functional recovery after mesenchymal stem cell administration}

The study by Gutiérrez-Fernández and colleagues [1] shows that, in comparison with the controls, the administration of MSCs significantly improved the neural function, reduced apoptotic cell death, increased cell proliferation, and reduced reactive gliogenesis that causes scar formation.

Several mechanisms are proposed to explain the successful improvement of neural functional recovery through MSC administration: (a) exogenous tissue repair through migration/integration, (b) neuroprotection through the reduction of cell death or immunomodulation, (c) enhancement of endogenous tissue repair, and (d) plasticity promotion in synapse formation [1,3,5]. Brain tissue consists of a major functional unit (that is, the neurovascular unit) that contains neural and vascular cells. Thus, to achieve a more effective tissue reconstruction, both cell lineages need to be considered. Previous studies have shown that MSCs differentiate into neurons, glial cells, and vascular endothelial cells, in both in vitro and in vivo conditions [4,5,7-10]. However, in the related article and several other reports, there was no graft after the transvenous MSC administration, even though the immunological feature of MSCs can prevent allograft rejection $[1,8]$. Therefore, the proposed cure mechanisms would be associated with the trophic factors secreted from MSCs. Correspondingly, the authors observe elevated levels of neurogenesis, synaprogenesis, oligodendrogenesis, and angiogenesis markers with increased levels of soluble factors in peri-infarct tissue 14 days after treatment with MSCs [1]. Interestingly, the findings indicate that both MSCs administered just 30 minutes after ischemia can maintain the therapeutic effects on the brain repair process even 14 days later. In all comparative parameters, the present study shows no significant difference between the group that received BM-MSCs and the one that received AD-MSCs [1], whereas some reports indicate that each set of MSCs has different abilities in terms of secreting trophic factors $[8,10]$. It is difficult to define the perfect integrity of each set of MSCs. In the most popular protocol, MSCs are currently isolated with a few cell surface markers $[4,5]$. The different results are expected to be verified by further findings about cell quality and integrity.

Nevertheless, the multipotency of MSCs would surely be beneficial if the MSCs were to integrate into the target.
The previous study by Gutiérrez-Fernández and colleagues [9] and other reports indicate that transarterial administration would deliver MSCs to the peri-infarct area [3], implying the possibility of application to neuroendovascular therapies through a catheter. The neural induction potential of MSCs has been extensively examined [5]. Still, further analyses are expected to be conducted on gene regulation mechanisms, including epigenetics while neural differentiation is occurring. In this context, an analysis of RNA and microRNA profiles was recently reported [11]. Also, in vitro comparative studies on the molecular biological characteristics have begun to be published, and differences among the various MSCs (BM-MSCs, AD-MSCs, and so on) have been detected $[10,12]$. The development of in vitro analysis will contribute to customizing the selection of appropriate graft MSCs in accordance with individual conditions.

\section{The role of in vivo and clinical studies in finding an ideal cell delivery}

For the best therapy, the maximum therapeutic effects should be provided to patients with a protocol that is minimally invasive and provides the least amount of suffering and risk. That is the final goal for translational research. The comparative study by Gutiérrez-Fernández and colleagues [1] has demonstrated that both sets of major MSCs - BM-MSCs and AD-MSCs - could become effective tools via intravenous administration, which is the least invasive route. The authors also have indicated that there is a need to study acute-phase treatment with allogenic grafts by showing the long-term effects on brain repair after a single injection of MSCs just after onset [1]. Given the results of several successful clinical trials and the theoretical advantages of MSC therapy, it is hoped that this therapy can become a reliable treatment $[3,4,7]$. Hence, further comparative studies are desired to assess the protocols in both the preclinical and clinical stages. While the development of an ideal treatment requires huge efforts and the continual accumulation of major findings, such as the work of Gutiérrez-Fernández and colleagues [1,9], careful studies on multiple aspects will contribute to achieving the best therapy.

\section{Abbreviations}

AD-MSC, adipose tissue-derived mesenchymal stem cell; BM-MSC, bone marrow-derived mesencymal stem cell; MSC, mesenchymal stem cell.

\section{Competing interests}

The author declares that she has no competing interests.

\section{Author details}

'Department of Cell Signaling, Gifu University Graduate School of Medicine, 1-1 Yanagido, Gifu 5011194, Japan. ${ }^{2}$ Department of Neurosurgery, Gifu University Graduate School of Medicine, 1-1 Yanagido, Gifu 5011194, Japan.

Acknowledgments

This work was supported in part by the Japan Society for the Promotion of Science (JSPS KAKENHI grant number 24700824 to the author). The author 
is grateful to Manfred Biebl and Shin-ichiro Hayashi for editing support and to Shigeru Nakashima (a chair of the Department of Cell Signaling, Gifu

University) and Toru Iwama (a chair of the Department of Neurosurgery, Gifu

University) for general support.

Published: 31 January 2013

\section{References}

1. Gutiérrez-Fernández M, Rodríquez-Frutos B, Ramos-Cejudo J, VallejoCremades MT, Fuentes B, Cerdán S, Díez-Tejedor E: Effects of i.v. administration of allogenic bone marrow- and adipose tissue-derived mesenchymal stem cells on functional recovery and brain repair markers in experimental ischemic stroke. Stem Cell Res Ther 2013, 4:11.

2. Burns TC, Steinberg GK: Stem cells and stroke: opportunities, challenges and strategies. Expert Opin Biol Ther 2011, 11:447-461.

3. LiY, Chopp M: Marrow stromal cell transplantation in stroke and traumatic brain injury. Neurosci Lett 2009, 456:120-123.

4. Mizuno H, Tobita M, Uysal AC: Concise review: adipose-derived stem cells as a novel tool for future regenerative medicine. Stem Cells 2012, 30:804-810.

5. Maltman DJ, Hardy SA, Przyborski SA: Role of mesenchymal stem cells in neurogenesis and nervous system repair. Neurochem Int 2011, 59:347-356.

6. Jorgensen C: Mesenchymal stem cells immunosuppressive properties: is it specific to bone marrow-derived cells? Stem Cell Res Ther 2010, 1:15.

7. Honmou O, Onodera R, Sasaki M, Waxman SG, Kocsis JD: Mesenchymal stem cells: therapeutic outlook for stroke. Trends Mol Med 2012, 18:292-297.
8. Ikegame Y, Yamashita K, Hayashi S, Mizuno H, Tawada M, You F, Yamada K Tanaka Y, Egashira Y, Nakashima S, Yoshimura S, Iwama T: Comparison of mesenchymal stem cells from adipose tissue and bone marrow for ischemic stroke therapy. Cytotherapy 2011, 13:675-685.

9. Gutiérrez-Fernández M, Rodríguez-Frutos B, Álvarez-Grech J, VallejoCremades MT, Expósito-Alcaide M, Merino J, Roda JM, Díez-Tejedor E: Functional recovery after hematic administration of allogenic mesenchymal stem cells in acute ischemic stroke in rats. Neuroscience 2011, 175:394-405

10. Zhang HT, Liu ZL, Yao XQ, Yang ZJ, Xu RX: Neural differentiation ability of mesenchymal stromal cells from bone marrow and adipose tissue: a comparative study. Cytotherapy 2012, 14:1203-1214.

11. Crobu F, Latini V, Marongiu MF, Sogos V, Scintu F, Porcu S, Casu C, Badiali M, Sanna A, Manchinu MF, Ristaldi MS: Differentiation of single cell derived human mesenchymal stem cells into cells with a neuronal phenotype: RNA and microRNA expression profile. Mol Biol Rep 2012, 39:3995-4007.

12. Abu Kasim NH, Govindasamy V, Gnanasegaran N, Musa S, Pradeep PJ, Srijaya TC, Aziz ZA: Unique molecular signatures influencing the biological function and fate of post-natal stem cells isolated from different sources. $J$ Tissue Eng Regen Med 2012 Dec 10. [Epub ahead of print].

doi:10.1186/scrt157

Cite this article as: Ikegame $Y$ : Among mesenchymal stem cells: for the best therapy after ischemic stroke. Stem Cell Research \& Therapy 2013, 4:9. 